\title{
One small wearable, one giant leap for patient safety?
}

\author{
Frederic Michard ${ }^{1}\left[\right.$ [D $\cdot$ Robert H. Thiele ${ }^{2} \cdot$ Morgan Le Guen $^{3}$
}

Received: 23 September 2021 / Accepted: 12 October 2021 / Published online: 19 October 2021

(c) The Author(s), under exclusive licence to Springer Nature B.V. 2021

Most in-hospital adverse events requiring the intervention of a rapid response team and/or ICU admission do not happen out of the blue. They are frequently preceded by a progressive clinical decline that may be overlooked for hours because of the intermittent nature of vital sign spot-checks on general hospital wards [1]. For example, on occasion a nurse may spend only 10 min every $4 \mathrm{~h}$ with a patient, which would account for only $4 \%$ of the time he or she is on the wards. The National Institute for Clinical Excellence (NICE) in the UK claimed that "Patients who are admitted to hospital believe that they are entering a place of safety. They feel confident that, should their condition deteriorate, they are in the best place for prompt and effective treatment. Yet there is evidence to the contrary" (https://www.nice.org.uk/ guidance/CG50). Indeed, a recent study [2] demonstrated that around $80 \%$ of hypoxemic and hypotensive events occur between intermittent spot-checks and are therefore detected with a delay, if not entirely missed. Therefore, the early detection of clinical deterioration might be a major opportunity to improve patient safety on hospital wards $[1,3]$.

At home, when outpatients with chronic disease start to deteriorate, symptoms are not always overt, and patients may wait days, if not weeks, before getting in touch with a medical professional. Of course, they may seek advice by telephone, but it is very challenging for a clinician to fully appreciate the situation without seeing the patient and without having access to objective data, including an assessment of vital signs. This is the reason why virtual-visits or teleconsultations and home monitoring are on the rise.

When Neil Armstrong first walked on the moon in 1969, his ECG was continuously monitored wirelessly (Fig. 1).

Frederic Michard

frederic.michard@bluewin.ch

1 MiCo, Denens, Switzerland

2 Department of Anesthesiology, University of Virginia School of Medicine, Charlottesville, VA, USA

3 Department of Anesthesiology and Pain Medicine, Hôpital Foch, University Versailles Saint Quentin, Suresnes, France
Since then, both rocket science and wearable technologies have made tremendous progress. Today, SpaceX, Virgin Galactic and Blue Origin are private companies able to launch their own space vessels. Multiple startups and medical device companies have also launched - not in space but on the market-mobile solutions for the remote, automatic, and continuous monitoring of vital signs. These new monitoring solutions are briefly discussed in the light of recent articles published in the Journal of Clinical Monitoring and Computing.

\section{Continuous monitoring on hospital wards}

General hospital wards are characterized by a low nurse-topatient ratio and recent studies have shown that scheduled vital sign spot-checks may be omitted $33 \%$ of the time and even when taken, may be incomplete in $25 \%$ of the cases [4]. Respiratory rate (RR) is frequently missing when not simply guesstimated to be around 16 or 20 breaths $/ \mathrm{min}$. This is problematic because tachypnea is common during stress, pain, respiratory distress, metabolic disorders, sepsis, and $\mathrm{RR}$ is therefore a very sensitive marker of clinical deterioration [5].

In this issue, Tanaka et al. [6] investigated the potential value of a new contact-free sensor designed to monitor RR. The monitoring system was a microwave Doppler mounted on the ceiling of the patient room. Tanaka et al. [6] compared measurements of RR by this new contact-free monitoring system to visual counting done by experienced nurses over a $60 \mathrm{~s}$ period. The accuracy (or bias) was comparable to what was obtained with the widely used tethered impedance pneumography method. However, the new Doppler method was not as precise as impedance pneumography (as illustrated by wider limits of agreement) and the sensitivity to detect tachypnea was too low (50\%) to envision clinical implementation.

In the same issue, Järvelä et al. [7] investigated the accuracy and precision of another innovative RR monitoring system. The system was a wireless impedance pneumographic 


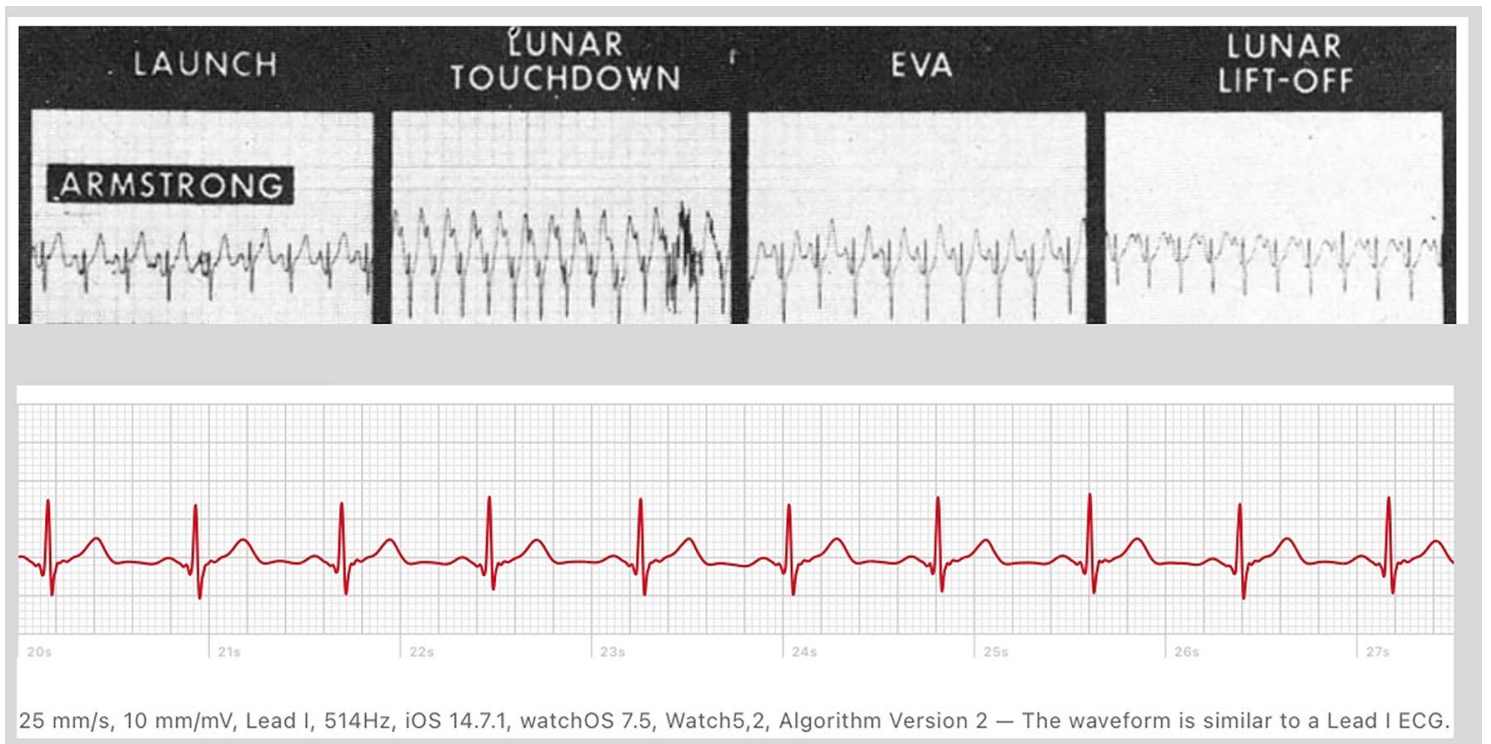

Fig. 1 ECG monitoring. Top: The inception of remote monitoring with the ECG recording of Neil Armstrong during the Apollo 11 mission. Bottom: Today, everyone can self-record ECG leads with a smartwatch (example from one of the authors)

sensor made of three surface skin electrodes connected, via a proprietary connectivity protocol, to a smartphonelike gateway. When compared with reference capnographic measurements, $>99 \%$ of RR wireless measurements were in the safe zones (A \& B) of the Clarke error grid. In addition, the new sensor had a sensitivity of $81 \%$ and a specificity of $93 \%$ to detect tachypnea. These findings are in contrast with the disappointing results of previous studies assessing the performance of wireless adhesive patches designed to monitor RR from ECG respiratory variations and accelerometer data [8].

Over the last few years, many systems have received regulatory clearance to continuously monitor vital signs [9]. They include numerous wireless pulse oximeters, piezoelectric sensors to be put under the bed mattress (EarlySense from Israel), thoracic adhesive patches (BioBeat from Israel, Isansys and Sensium from the UK, LifeSignals, PMD Solutions, VitalConnect, Vitls, and Vivalink from the USA), finger blood pressure cuffs (Caretaker medical from USA), and bioimpedance necklaces (Tosense from USA, CloudDX from Canada). Sensors can also be combined to gather additional information. For instance, the simultaneous recording of an ECG lead from thoracic skin surface electrodes and a peripheral pulse oximetry waveform enables the measurement of the pulse wave transit time (aka pulse arrival time) and hence a continuous estimation of blood pressure (Sotera from USA). In the future, the miniaturization of the volume clamp method into a finger ring (CNSystems from Austria) or the prediction of blood pressure from a pulse oximetry waveform with machine learning algorithms (Valencell from USA) may also be useful to compute vital signs.

\section{Home monitoring}

The coronavirus 2019 (COVID) pandemic has been a catalyst for the development and adoption of home monitoring solutions [10]. Telemedicine and home monitoring are obviously useful to avoid physical contact with potentially contagious patients. They may also be useful to avoid hospital admission or after early discharge from the emergency department or hospital wards [11]. Many nonCOVID patients may benefit from remote monitoring and management as well, in particular cancer patients treated by chemotherapy, patients discharged from hospital after a major surgery, and patients with chronic disease at high risk of hospital re-admission. In patients with congestive heart failure, the remote monitoring of pulmonary artery pressure with an implantable micro-electromechanical system (aka MEMS) has been shown to be useful to adjust therapy and prevent hospital readmissions [12]. Whether similar benefits could be reported with non-invasive bioimpedance necklaces able to detect early changes in thoracic fluid content remains to be determined [13].

There are two ways to envision home monitoring. The first relies on patients to self-monitor their own vital signs from time to time (Fig. 2). It is now easy to find and buy wireless medical grade products at a reasonable price and to use smartphones to store and share the recorded physiologic information [14]. Some companies offer vital sign "toolboxes" containing devices necessary to measure vital signs (usually a brachial cuff, a pulse oximeter and a thermometer). When patients detect abnormalities, they can 


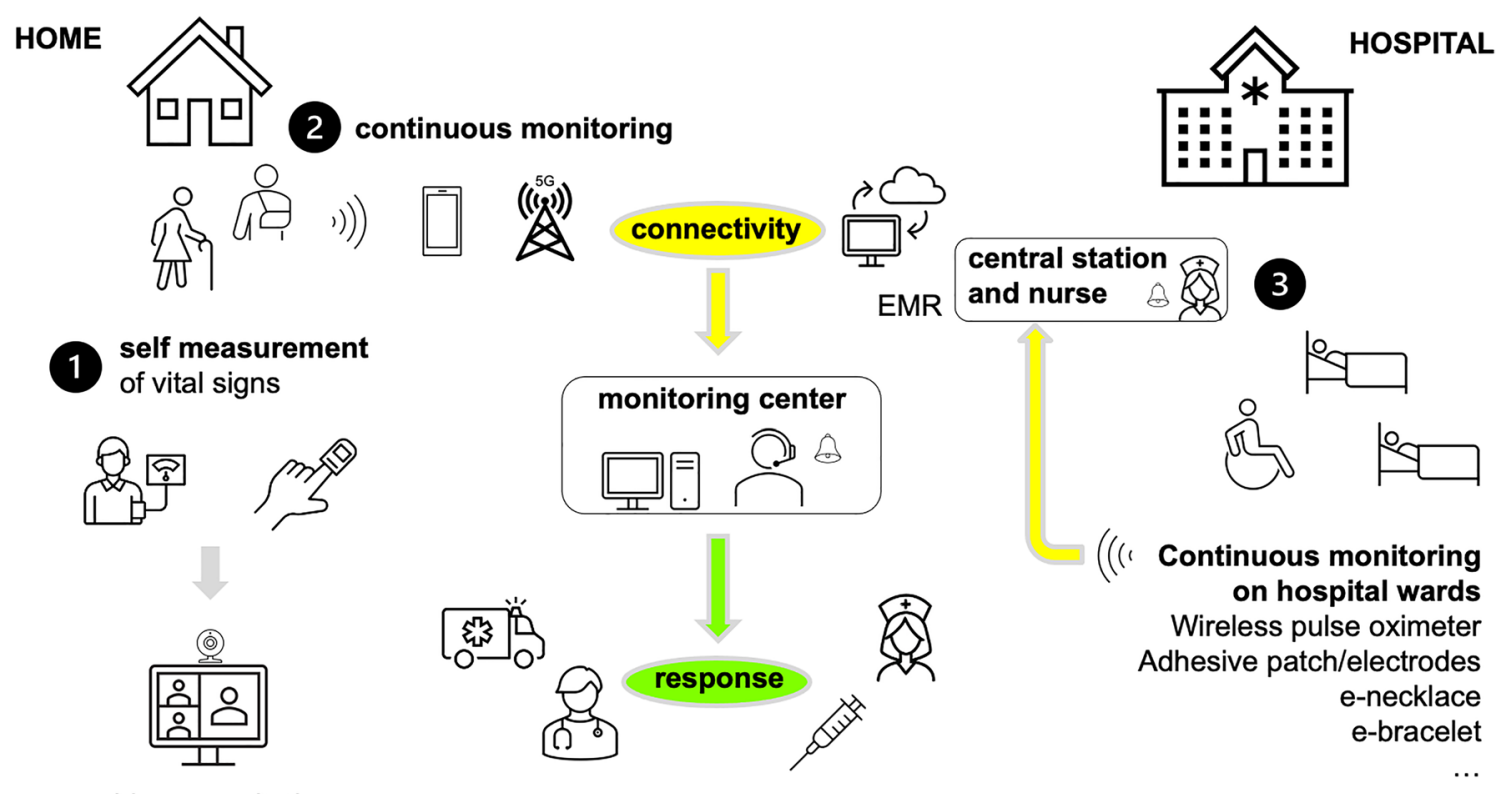

video consultation

Fig. 2 Remote detection of clinical deterioration for in- and outpatients. From home, patients can self-measure selected vital signs (1) and, when needed, share them during a video consultation. They can also wear one or more sensors for continuous monitoring of selected vital signs (2), and the information is processed by a monitoring center. On hospital wards, patients at high-risk of clinical deterioration are continuously monitored (3), and the alerts are received by the nurse, at a central station and/or in a monitoring or command center. Robust connectivity (yellow) and appropriate response (green) are as important as the sensor itself share the information remotely or during a video-consultation with their general practitioner, oncologist, cardiologist, or pulmonologist. The use of embedded cameras, present in computers, tablets, or smartphones, has also been proposed to capture vital signs during video consultations. In this issue, Pham et al. [15] meta-analyzed twenty-two studies investigating the use of consumer grade webcams to estimate vital signs. They concluded that webcams may be useful to measure heart rate (from subtle changes in color face invisible to the human eye), but not yet to estimate respiratory rate, blood pressure and oxygen saturation.

The alternative to self-monitoring is to ask patients to wear one or more sensors for days or weeks. The recorded physiologic information can then be automatically sent via a gateway (e.g. the patient's smartphone) to a monitoring center where a dedicated staff analyzes and interprets the information and decides what should be the appropriate response (e.g. recommend a consultation or send over a nurse, or an ambulance if a transfer to the hospital appears to be the best option). In any case, and as illustrated in Fig. 2, the sensor is not the only link of the patient safety chain. Connectivity and the response to the alert are as important and may be real logistical challenges for the widespread and successful implementation of remote monitoring solutions.

\section{Conclusion}

New methods are emerging to monitor vital signs remotely on both hospital wards and at home. For inpatients, automatic and continuous monitoring systems may unload nurses from time-consuming and repetitive tasks, detect clinical deterioration at an early stage, and decrease the number of emergency interventions, ICU admissions, and cardiac arrests. For outpatients with chronic disease (e.g. patients with congestive heart failure), remote monitoring has potential for the timely recognition of exacerbation, earlier initiation of therapeutic adjustments and prevention of hospital re-admissions. For patients undergoing major surgery, home monitoring may help before hospital admission (e.g. during pre-habilitation programs) and after hospital discharge for the early detection of post-operative complications. Thanks to the digital revolution, the pace of monitoring innovations is accelerating. As clinicians, it is now our role and responsibility to conduct studies to validate new monitoring systems [16], to assess their impact on clinical outcomes and health care costs, and to clarify which patients may benefit the most.

Author contributions FM drafted the manuscript and all authors worked on and approved the final version. 
Funding Not applicable.

\section{Declarations}

Conflict of interest FM is the founder and managing director of MiCo, a Swiss consulting and research firm. RHT and MLG have nothing to declare in relation to this editorial.

Consent to participate Not applicable.

Ethical approval Not applicable.

\section{References}

1. Michard F, Kalkman C. Rethinking patient surveillance on hospital wards. Anesthesiology. 2021;135:531-40.

2. Saab R, Wu BP, Rivas E, et al. Failure to detect ward hypoxaemia and hypotension: contributions of insufficient assessment frequency and patient arousal during nursing assessments. Br J Anaesth. 2021;127:760-768.

3. Bates DW, Zimlichman E. Finding patients before they crash: the next major opportunity to improve patient safety. BMJ Qual Saf. 2015;24:1-3.

4. Eddahchouri Y, Koeneman M, Plokker M, Brouwer E, van de Belt $\mathrm{TH}$, van Goor H, Bredie SJ. Low compliance to a vital sign safety protocol on general hospital wards: a retrospective cohort study. Int J Nurs Stud. 2021;115:103849.

5. Akel MA, Carey KA, Winslow CJ, et al. Less is more: detecting clinical deterioration in the hospital using machine learning using only age, heart rate and respiratory rate. Resuscitation. 2021;168:6-10.

6. Tanaka H, Yokose M, Takaki S, et al. Evaluation of respiratory rate monitoring using a microwave Doppler sensor mounted on the ceiling of an intensive care unit: a prospective observational study. J Clin Monit Comput. 2021. https://doi.org/10.1007/ s10877-021-00733-w.

7. Jarvela K, Takala P, Michard F, Vikatmaa L. Clinical evaluation of a wearable sensor for mobile monitoring of respiratory rate on hospital wards. J Clin Monit Comput. 2021. https://doi.org/10. 1007/s10877-021-00753-6.

8. Breteler MJM, KleinJan EJ, Dohmen DAJ, et al. Vital signs monitoring with wearable sensors in high-risk surgical patients: a clinical validation study. Anesthesiology. 2020;132:424-39.

9. Michard F, Bellomo R, Taenzer A. The rise of ward monitoring: opportunities and challenges for critical care specialists. Intensive Care Med. 2019;45:671-3.

10. Michard F, Shelley K, L'Her E. COVID-19: pulse oximeters in the spotlight. J Clin Monit Comput. 2021;35:11-4.

11. Shah S, Majmudar K, Stein A, et al. Novel use of home pulse oximetry monitoring in COVID-19 patients discharged from the emergency department identifies need for hospitalization. Acad Emerg Med. 2020;27:681-92.

12. Radhoe SP, Veenis JF, Brugts JJ. Invasive devices and sensors for remote care of heart failure patients. Sensors. 2021;21:2014.

13. Michard F. A sneak peek into digital innovations and wearable sensors for cardiac monitoring. J Clin Monit Comput. 2017;31:253-9.

14. Michard F. Toward smart monitoring with phones, watches, and wearable sensors. Anesthesiol Clin. 2021;39:555-64.

15. Pham C, Poorzargar K, Nagappa M, et al. Effectiveness of consumer grade contactless vital signs monitors: a systematic review and meta-analysis. J Clin Monit Comput. 2021. https://doi. org/10.1007/s10877-021-00734-9.

16. Le Guen M, Squara P, Ma S, et al. Patch validation: an observational study protocol for the evaluation of a multisignal wearable sensor in patients during anaesthesia and in the postanesthesia care unit. BMJ Open. 2020;10:e040453.

Publisher's Note Springer Nature remains neutral with regard to jurisdictional claims in published maps and institutional affiliations. 